Journal of the Egyptian Society of Parasitology, Vol.43, No.2, August 2013 J. Egypt. Soc. Parasitol., 43(2), 2013: 351 - 372

\title{
INFECTION CONTROL TO AVOID SURGICAL SITE INFECTION
}

\section{By}

\author{
MOSTAFA H. ABOU-HATAB ${ }^{1}$ and MAMDOUH M. EL-BAHNASAWY ${ }^{2}$ \\ Consultant of General Surgery ${ }^{1}$ and Consultant of Endemic Diseases and \\ Fevers $^{2}$, Military Medical Academy

\section{Abstract}

Infection control is a discipline that applies epidemiologic and scientific principles and statistical analysis to prevent or reduce rates of nosocomial infections. Effective infection control programs proved to reduce the rates of nosocomial infections and to be cost-effective. It is a key component of the broader discipline of hospital epidemiology. As an example, the anesthesia team acts every day in a highly complex and high risk environment for the transmission of pathogenic organisms and induction of infectious complication. In order to achieve the main goal of preventing or reducing the risk of hospital-acquired infections, a hospital epidemiology program should have the following oversight functions and responsibilities: Surveillance, either hospital-wide or targeted Education about prevention of infections. Outbreak investigations cleaning, disinfection and sterilization of equipments and disposal of infectious waste hospital employee health, specifically after exposure to either blood-borne or respiratory pathogens, Review of antibiotic utilization and its relationship to local antibiotic resistance patterns, Prevention of infections due to percutaneous intravascular devices. Development of infection control policies and procedures oversight on the use of new products that directly or indirectly relate to the risk of nosocomial infections.

Key words: Hospital, Surgery, Infection control, Site infection, Management.

\section{Review and Management}

Surgical wound infections are the second most common nosocomial infection. Although usually localized to the incision site, surgical wound infections can also extend into adjacent deeper structures; thus, the term surgical wound infection has now been replaced with the more suitable name, surgical site infection (SSI).

\section{Hospital Control measures:}

The CDC has developed criteria for defining SSIs, which have become the national standard and are widely used by surveillance and surgical personnel.
These criteria define SSIs as infections related to the operative procedure that occur at or near the surgical incision (incisional or organ/space) within 30 days of an operative procedure or within one year if an implant is left in place (CDC, 1992a).

Among surgical patients SSIs are the most common nosocomial infection; they account for $38 \%$ of nosocomial infections. It is estimated that SSIs develop in 2 to $5 \%$ of the more than 30 million patients undergoing surgical procedures each year; one out of every 
24 patients who have inpatient surgery in the United States has a postoperative SSI (CDC, 1992b). The cost of SSIs is substantial. One study found an average total cost of $\$ 5155$ during the eight week period after discharge for patients with an SSI diagnosed after discharge compared to $\$ 1773$ for those without an SSI (Perencevich et al, 2003).

The Centers for Medicare \& Medicaid Services, in collaboration with the CDC, has implemented the surgical infection prevention project to decrease the morbidity and mortality associated with postoperative surgical site infections. So, surgical care improvement project, a national quality partnership of organizations committed to improving the safety of surgical care, was announced (Bratzler and Hunt, 2006).

\section{General practices:}

The most important factors in the prevention of SSIs are: The general health of the patient meticulous operative techniques Timely administration of effective preoperative antibiotics

A number of practices and policies have been used over the years to reduce the risk of SSIs, including: Preoperative showering with antimicrobial soaps Application of antiseptics to the skin of the patient Washing and gloving of the surgeon's hands Use of sterile drapes Use of gowns and masks by operating room personnel (Mangram et al, 1999).

Most of these rituals were developed to reduce contact with flora from the hands, hair, scalp, nares, and oropharynx of hospital personnel, which were believed to be the source of microorganisms causing SSIs. However, care- fully controlled studies have failed to clearly show the benefits of most of these rituals.

Modern methods of antisepsis can reduce, but not eliminate, the skinassociated bacteria of the surgical patient; approximately $20 \%$ of the bacteria are located in hair follicles and sebaceous glands, which are not reached with preoperative antiseptic agents (Selwyn and Ellis, 1972). Designed studies showed that whole-body disinfection through preoperative showers or local antisepsis through skin preparation does not prevent SSIs (Cruse and Ford, 1980). A meta-analysis of six trials involving a total of 10,007 participants came to the same conclusion: there is no evidence of benefit for preoperative showering or bathing with an antiseptic preparation over other wash products to reduce SSIs (Hayek et al, 1987).

A related issue, the value of skin antiseptics prior to clean surgery, was evaluated in a systematic review (Rotter et al, 1988). They concluded that there was insufficient evidence to support or refute the use of skin antiseptics in this setting or of one antiseptic over another.

Similar to antiseptics, the use of barrier devices (masks, caps, gowns, drapes, and shoe covers) to prevent SSIs is not supported by rigorously controlled and valid clinical studies. The primary role for these barrier devices is to protect operating room personnel from exposure to infectious blood or body fluids. Currently, the Occupational Health and Safety Administration (OSHA) guidelines require 
that masks, caps, and shoe covers should be worn when gross contamination can be reasonably anticipated, but in fact most operating rooms in the United States require universal use of such gear regardless of the type of procedure (Webster and Osborne, 2006).

Good surgical technique is fundamental to lowering SSI rates. These techniques include: gentle traction, effective hemostasis, removal of devitalized tissues, obliteration of dead space, irrigation of tissues by saline during long procedures to avoid excessive drying, use of fine, non-absorbed monofilament suture material, judicious use of closed suction drains, and wound closure without tension (Edwards et al, 2004).

\section{Choice of surgical hand hygiene:}

Although no randomized, controlled trials have been conducted to indicate that SSI rates are substantially lower with preoperative scrubbing, preoperative cleansing of hands and forearms with an antiseptic agent has been an accepted practice. Hand-rubbing with aqueous alcoholic solution may be as effective as traditional hand-scrubbing with antiseptic soap to prevent surgical site infections (Altemeier et al, 1984).

A randomized trial involving six surgical services from teaching and nonteaching hospitals in France compared two hand-cleansing methods alternately every other month: a hand-rubbing protocol with $75 \%$ aqueous alcoholic solution containing propanol-1, propanol-2, and mecetronium etilsulfate; and a hand-scrubbing protocol with antiseptic preparation containing 4 percent povidone iodine or $4 \%$ chlorhexidine glu- conate. Surgical site infection rates were 55 of $2252(2.44 \%)$ in the handrubbing protocol and 53 of 2135 $(2.48 \%)$ in the hand-scrubbing protocol. The hand-rubbing protocol was better tolerated by the surgical teams and improved compliance (44\% versus $28 \%$ ) with hygiene guidelines (Tanner et al, 2008).

Guidelines support using either an antimicrobial soap or an alcohol-based hand rubs with persistent activity for surgical hand antisepsis (Parienti et al, 2002).

\section{Antimicrobial prophylaxis:}

The goal of antimicrobial prophylaxis is to eradicate or retard the growth of endogenous microorganisms. Optimal prevention of SSIs requires that the antimicrobial prophylaxis be combined with other measures described below.

The efficacy of antibiotic prophylaxis in clean and clean-contaminated surgery has been clearly established. Patients who receive prophylactic antibiotics within a two hour "window" before the initial incision have lower rates of SSI than patients who receive antibiotics either too early or postoperatively (Boyce and Pittet, 2002).

Despite evidence of effectiveness and the publication of guidelines for antimicrobial prophylaxis to prevent SSIs, substantial inconsistencies occur in the use of prophylactic antimicrobials for patients undergoing major surgery. Suboptimal use of antimicrobial prophylaxis was examined in a systematic random sample of 34,133 Medicare inpatients undergoing surgical procedures in 2965 acute care hospitals in the USA by National Surgical Infection 
Prevention Project (van Kasteren et al, 2007). Antimicrobial agents consistent with published guidelines were administered to $93 \%$ of the patients. An antimicrobial dose was administered to only $56 \%$ of patients within one hour before incision. Antimicrobial prophylaxis was discontinued within 24 hours of surgery in only $41 \%$ of patients (Bratzler et al, 2005).

A subsequent collaborative study involving 56 hospitals in 50 states tested various "improvement methodologies" and demonstrated that simple programs could produce improvement in the rates of compliance with the above three measures. As an example, the mean rate of compliance with optimal timing of preoperative antimicrobial prophylaxis rose to $95 \%$ and antimicrobial prophylaxis was discontinued within one hour following surgery in $85 \%$ of patients (Dellinger et al, 2005). Most importantly, hospitals that participated in the pilot study reported a mean $27 \%$ reduction in their rate of SSIs (from 2.3 to $1.7 \%$ in the first versus last three months of the study). But, the most clean surgeries do not need antimicrobial prophylaxis unless there is a high risk infection or the consequences of a surgical site infection are disastrous (e.g., CABG, insertion of a prosthesis or laminectomy). Also, since most contaminated or dirty wounds require a therapeutic course of antibiotics, antimicrobial therapy in such patients is actually therapeutic rather than prophylactic (Bolon et al, 2004).

\section{Antibiotic selection:}

There are minor differences between published guidelines regarding appro- priate agents for antimicrobial prophylaxis; following general recommendations for intravenous (IV) antimicrobial prophylaxis prior to surgery reasonable and appropriate are considered (Garey et al, 2008). The first generation cephalosporin cefazolin (1 to $2 \mathrm{~g} I V$ ) proved effective for most clean procedures because of its activity against many staphylococci and streptococci. The second generation cephalosporin cefuroxime (1.5 g IV) can be given instead of cefazolin in thoracic (cardiac and noncardiac) and orthopedic procedures. For procedures that might involve exposure to bowel anaerobes (including Bacteroides fragilis), the second generation cephalosporins cefoxitin (1 to $2 \mathrm{~g} \mathrm{IV}$ ) or cefotetan are more effective than cefazolin against these organisms. However, many bowel aerobic gram-negative bacilli such as E. coli have become resistant to cefoxitin. Thus, alternative regimens such as cefazolin (1 to $2 \mathrm{~g}$ IV) plus metronidazole $(0.5 \mathrm{~g}$ IV) or monotherapy with ampicillin/sulbactam (3 g IV) are reasonable alternatives. Most patients with a penicillin allergy can be treated with cefazolin, since allergic reactions to cephalosporins are infrequent except in patients with severe IgE-mediated reactions to penicillin, some of whom have experienced anaphylaxis upon first exposure to cephalosporins (Finkelstein et al, 2002). When allergy prevents administration of a cephalosporin, alternatives include I.V. vancomycin ( $1 \mathrm{~g}$ [10-15 mg/kg]) or clindamycin $(600-$ $900 \mathrm{mg}$ ). Many would add a drug to cover gram-negative bacteria, as gentamicin, ciprofloxacin, levofloxacin, or 
aztreonam, particularly in patients undergoing a colorectal procedure, hysterectomy, or vascular surgery involving an incision in groin. Local resistance patterns should be taken into account in patients with a long preoperative hospitalization (Vuorisalo et $a l, 1998)$. Vancomycin can be given in institutions where methicillin-resistant $S$. aureus or methicillin-resistant coagulase-negative staphylococci cause surgical site infections (Pons et al, 1993).

\section{Role of vancomycin:}

There is an increasing role for vancomycin in surgical prophylaxis, although there are few data to assess the efficacy of this strategy. It may have a role in the following circumstances: Prevention of methicillin-resistant $S$. aureus (MRSA) infection in centers with a high prevalence of surgical infections due to this organism reduction in the risk of methicillin-resistant coagulase-negative staphylococcal and enterococcal infection following prosthetic heart valve or prosthetic vascular graft implantation Prevention of infection in patients who have received broad-spectrum antimicrobial therapy

Despite these considerations, randomized trials have failed to demonstrate a benefit of vancomycin compared to cefazolin (cefuroxime or of teicoplanin compared to cefazolin ceftizoxime (Saginur et al, 2000).

Equivalent efficacy for vancomycin and beta-lactams was noted in a metaanalysis of seven randomized trials of 5761 procedures. In subset analyses, beta-lactam prophylaxis was significantly more effective for prevention of chest SSIs with a strong trend toward a benefit for prevention of deep chest SSIs and SSIs caused by gram-positive bacteria. On the other hand, there was a trend toward glycopeptides being superior for prevention of leg SSIs or SSIs caused by methicillin-resistant grampositive bacteria. The authors concluded that beta-lactams should continue to be the standard prophylaxis after cardiac surgery in most patients (Periti et al, 1999). Nonetheless, vancomycin is often used in institutions with high rate of MRSA infections. even though guidelines do not define minimum criteria. Guidelines from the Surgical Care Improvement Project do state that vancomycin is an acceptable antibiotic for patients undergoing cardiac, vascular, and orthopedic procedures. It is considered the vancomycin as appropriate only for selected patients even in hospitals where postoperative MRSA infections are common. Practice add vancomycin as preoperative prophylaxis in patients undergoing high-risk surgeries with identifiable risk factors for postoperative MRSA infection, as recent hospitalized, renal disease, or diabetes. So, add a beta-lactam antibiotic (cefazolin or cefuroxime) to provide appropriate coverage for gram-negative organisms. Alternatives in patients allergic to cephalosporins include gentamicin, ciprofloxacin, levofloxac in, or aztreonam (Bratzler and Houck, 2002).

\section{Prophylaxis timing:}

Guidelines from the medical letter and the Surgical Care Improvement Project recommend that antimicrobial therapy be administered within an hour prior to the surgical incision to ensure adequate drug tissue levels at time of 
initial incision (within 2 hours if vancomycin or fluoroquinolone is used) to prevent antibiotic-associated reactions around anesthesia time of induction (Dellinger, 2007). Support comes from a prospective observational study of 2847 patients undergoing elective clean or clean-contaminated surgery. Lowest rate of SSI $(0.6 \%)$ was in 1708 patients $(60 \%)$ who received antimicrobial prophylaxis in 2 hours before incision. Rates of SSI were higher in patients who received prophylaxis more than 2 hours prior to incision, within 3 hours after incision, or more than 3 hours after incision $(3.8,1,4, \& 3.3 \%$, respectively).

A similar U-shaped curve was noted in a prospective study of 1922 patients undergoing elective total hip arthroplasty between 2000 and 2002, with a trend toward lower rates of SSI in the 1679 patients $(87 \%)$ who were given antimicrobial therapy within an hour before incision.

The better outcomes with preoperative antimicrobial therapy within one to two hours before incision may be explained by the following factors, each of which has been associated with lower rates of SSIs: The higher serum antimicrobial concentrations during surgery and measurable levels at the end of surgery (DiPiro et al, 1985), Adequate tissue antimicrobial concentrations at the time of incision and wound closure (Wong-Beringer et al, 1995).

Antimicrobial therapy administered immediately before surgery may be less effective than antimicrobial therapy given between 60 and 30 minutes before surgery. In a prospective obser- vational study including 3836 surgical patients, antimicrobial therapy given 0 to 29 minutes prior to surgery was less effective than comparable therapy administered between 30 to 59 minutes preoperatively (Weber et al, 2008). This effect was observed even after statistical adjustment for other confounding risk factors such as ASA score, duration of surgery and wound class. In another study involving 2048 patients undergoing cardiac bypass surgery, patients who received vancomycin infusions 0 to 15 minutes prior to the beginning of surgery had higher rates of postoperative infection than those who received vancomycin 16 to 60 minutes preoperatively. These studies suggest that adequate time should be allowed for complete antibiotic infusion and achievement of steady state kinetics before proceeding with the surgical procedure (Garey et al, 2006).

\section{Repeat dosing:}

The Medical Letter recommends a single intravenous antimicrobial dose for procedures lasting less than four hours. For procedures lasting more than four hours or when major blood loss occurs, redosing is indicated every one to two half-lives of the drug in patients with normal renal function (cefazolin every 2 to 5 hours, cefoxitin every 2 to 3 hours, cefuroxime every 3 to 4 hours, ampicillin/sulbactam every 2 to 4 hours, vancomycin every 6 to 12 hours, clindamycin every 3 to 6 hours, and metronidazole every 6 to 8 hours).

The potential value of intraoperative redosing for prolonged surgical procedures was illustrated in a retrospective study that evaluated the risk of SSIs in 
1548 patients who, after preoperative administration of cefazolin prophylaxis, underwent cardiac surgery lasting more than four hours; intraoperative redosing was performed in 30\% (Zanetti et al, 2001). The overall risk of SSI was similar among patients with and without intraoperative redosing (9.4 versus 9.3\%). However, the risk of SSI was significantly reduced by intraoperative redosing in procedures lasting more than 400 min (7.7 versus $16.0 \%$; adjusted OR 0.44, 95\% CI 0.23-0.86).

The guidelines disagree regarding the need for repeat antibiotic dosing during the postoperative period. Medical letter consultants have indicated that repeat dosing after wound closure was not necessary and might increase antimicrobial resistance. However, some experts recommend a second dose with open heart surgery when the patient is removed from cardiopulmonary bypass. In contrast, the Surgical Care Improvement Project recommends postoperative therapy with discontinuation within 24 hours after the end of most surgeries and within 48 hours after the end of cardiac surgery.

These recommendations are based upon both randomized trials and observational studies that failed to demonstrate any beneficial effects of longcourse prophylaxis regimens. In a systematic review of randomized trials, there was no difference in the rate SSI with single dose compared to multiple dose regimens given for less than or more than 24 hours, combined odds ratio 1.04 , 95\% CI 0.86-1.25 (McDonald et al, 1998). In addition to lack of protection against SSI, antimicrobial therapy has been associated with an increased risk of acquired antimicrobial resistance if given for more than 48 hours (Pollard et al, 1979) and of intravascular line infections and bacteremia if given for more than four days. Despite all of these observations, a report from a surgical intensive care unit between 1995 and 1997 found that $61 \%$ of prophylactic antibiotics were continued beyond 24 hours (Harbarth et al, 2000).

\section{Cardiac surgery:}

Cardiac surgery (e.g., coronary artery bypass, heart valve replacement) is primarily a clean surgery with the majority of patients having a low preoperative risk of infection (e.g., NNIS risk index of 0 or 1). Despite their infrequency, deep organ/space SSIs (e.g., mediastinitis and prosthetic valve endocarditis) are catastrophic complications associated with substantial morbidity and mortality.

The $S$. aureus and coagulase-negative staphylococci are the predominant pathogens responsible for SSIs in cardiac surgery. When the gram-negative bacilli (e.g., Escherichia coli, Klebsiella spp, Enterobacter spp, Proteus spp, and Pseudomonas spp) are responsible for SSIs, contamination of the chest usually occurs during the saphenous vein harvesting (Namias et al, 1999).

A meta-analysis of 28 studies conducted abroad concluded that antimicrobial prophylaxis is beneficial in cardiothoracic surgery (Farrington et al, 1985). Previous studies describing failures of first generation cephalosporins in preventing SSIs due to methicillinsensitive S. aureus (Kreter and Woods, 
1992) led to the second generation cephalosporins adoption (such as cefuroxime) as the preferred antimicrobial prophylaxis in many centers. However, subsequent clinical trials showed equivalent (Kernodle et al, 1990) or even lower rates of $S$. aureus SSIs (Curtis et al, 1993) with cefazolin compared to cefuroxime. Because of its greater intrinsic activity against staphylococci, narrower side effect profile and antimicrobial spectrum, and much lower cost, cefazolin is the prophylactic agent of choice as it is in the Medical Letter and Surgical Care Improvement Project. The drugs are given I.V. within 60 minutes before the first incision. Vancomycin and clindamycin are alternatives if allergy limits the use of a cephalosporin Pearkman et al, 1998). There is disagreement about the need for repeat dosing during and after cardiac surgery.

\section{Intravascular device placement:}

Although not well studied, antimicrobial prophylaxis is generally used before placement of permanent pacemakers, electrophysiologic devices, ventricular assist devices, ventriculoatrial shunts, and arterial patches. Dosing regimens are similar to those used in cardiac surgery (Ahmed et al, 2012).

\section{Noncardiac thoracic surgery:}

Antimicrobial prophylaxis is given routinely in thoracic surgery. The efficacy of this approach was illustrated in a randomized trial of 127 patients undergoing thoracic surgery who were assigned to a single preoperative dose of cefazolin (1 g) or placebo (Doebbeling et al, 1990). Cefazolin therapy was associated with a significant reduc- tion in wound infection (1.5 vs $14 \%$ ) but not in empyema or pneumonia.

The recommended antibiotics are cefazolin ( 1 to $2 \mathrm{~g} \mathrm{IV}$ ), cefuroxime (1.5 g IV), or, in selected patients, vancomycin ( $1 \mathrm{~g}$ [10 to $15 \mathrm{mg} / \mathrm{kg}$ ] IV). As mentioned above, the indications for vancomycin are previous colonization with MRSA, hospitals in which MRSA and/or coagulase-negative staphylococci are a common cause of postoperative infection, and allergy to penicillin groups or cephalosporins. Clindamycin is an alternative to vancomycin in patients with beta-lactam allergy.

\section{Colorectal surgery:}

Antimicrobial prophylaxis is recommended for procedures involving the colon or rectum, and coverage must be provided against enteric gram-negative bacilli, anaerobes, and enterococci.

A meta-analysis evaluated 147 randomized trials that compared different antimicrobial regimens. The surgical wound infection was generally defined as purulent discharge in the surgical wound, with or without a positive culture (Baddour et al, 2003).

The following findings were noted that the overall rate of surgical wound infection with prophylaxis was $11 \%$. There was no significant difference among the different effective regimens, although many trials were underpowered to detect a moderate difference. But, a number of regimens were inadequate, including doxycycline alone, piperacillin alone, metronidazole alone, and oral neomycin plus erythromycin. A single dose given immediately before surgery or short-term use was as effective as regimens that included 
long-term postoperative therapy. First generation cephalosporins were as effective as later generation cephalosporins.

Surgical Care Improvement Project and the 2009 medical letter guidelines recommended a variety of antimicrobial regimens in patients undergoing colorectal surgery. Antimicrobial prophylaxis can consist of an orally administered antimicrobial bowel preparation, a preoperative parenteral antimicrobial, or combination of both. Oral prophylaxis appears to be as effective as intravenous prophylaxis (Song and Glenny, 1998). In the US $>90 \%$ of colorectal surgeons who administer oral bowel preparations also give parenteral antibiotics prior to surgery (Lewis, 2002).

Recommended oral prophylaxis consists of neomycin plus erythromycin or neomycin plus metronidazole, initiated no more than 18 to $24 \mathrm{~h}$ before the operation, along with administration of a mechanical bowel preparation. The recommended dosing schedules are: Oral neomycin $(1 \mathrm{~g})$ plus oral erythromycin base (1 g), both given 19, 18, and 9 hours before surgery Oral neomycin (2 g) plus oral metronidazole (2 $\mathrm{g}$ ), both given 13 and 9 hours before surgery

The recommended dosing schedules were somewhat different with the two guidelines. The Surgical Care Improvement Project recommends cefoxitin, cefotetan, cefazolin plus metronidazole, or ampicillin-sulbactam: Cefoxitin or cefotetan, both 1 to $2 \mathrm{~g}$ (20 to $40 \mathrm{mg} / \mathrm{kg}$ ) IV preoperatively and continued at eight hour intervals for up to 24 hours after the end of surgery.
However, there is evidence for emergence of resistance of many gramnegative bacilli to cefotetan and cefoxitin. In a study involving 450 patients undergoing elective colorectal surgery who received prophylaxis with cefotetan, postoperative SSI was observed in $26 \%$ of cases. Cefotetan resistance was seen in $67 \%$ of bacterial isolates (Doebbeling et al, 1990). Cefazolin (1 $\mathrm{g}$ IV if less than $80 \mathrm{~kg}$ and $2 \mathrm{~g}$ IV if more than $80 \mathrm{~kg}$ initially and repeated for two doses postoperatively at eight hour intervals) plus metronidazole (15 $\mathrm{mg} / \mathrm{kg}$ IV first dose, $7.5 \mathrm{mg} / \mathrm{kg}$ subsequent doses given IV twice postoperatively at six hour intervals). Ampicillin-sulbactam, $3 \mathrm{~g}$ IV preoperatively and continued at six hour intervals for up to 24 hours after the end of surgery, Ertapenem, $1 \mathrm{~g}$ IV preoperatively as a single dose.

A randomized trial compared ertapenem, a long-acting carbapenem, to cefotetan in 901 patients undergoing elective colorectal surgery; a single dose was given within an hour before the first surgical incision (Zmora et al, 2003). The rate of surgical site infection was significantly lower with ertapenem (17 versus $26 \%$ ).

Itani et al, (1996) noted that the rate of SSI in the trial was significantly higher than the rates reported from National Nosocomial Infections Surveillance (NNIS) System (Sexton, 2006) or in the meta-analysis cited above, mean $11 \%)$. Two-thirds of the gram-negative pathogens isolated from patients treated with cefotetan were resistant to this drug, a possible reflection at least in 
part of widespread use for surgical prophylaxis (Aznar et al, 1991).

Ertapenem was approved for use as prophylaxis in colorectal surgery in 2006. However, the 2009 the medical letter guidelines advise against the routine administration of carbapenems for surgical prophylaxis because widespread use of these drugs may result in increased rates of resistance. This recommendation is accepted because carbapenems are currently the best available drugs for the treatment of serious infections caused by aerobic gramnegative organisms that are resistant to other widely used antimicrobial drugs (Trivedi et al, 2012).

A number of options were recommended for patients with beta-lactam allergy: Clindamycin (600 to $900 \mathrm{mg}$ IV and repeated twice postoperatively at eight hour intervals) plus either gentamicin $(1.5 \mathrm{mg} / \mathrm{kg}$ IV and repeated twice postoperatively at 8 to 12 hour intervals, or parenteral fluoroquinolone (ciprofloxacin $400 \mathrm{mg}$ IV and repeated once postoperatively at 12 hours or a single IV dose of levofloxacin $750 \mathrm{mg}$ or moxifloxacin $400 \mathrm{mg}$ ) Metronidazole $(15 \mathrm{mg} / \mathrm{kg}$ IV first dose, $7.5 \mathrm{mg} / \mathrm{kg}$ subsequent doses given IV twice postoperatively at six hour intervals) and aztreonam (1 to $2 \mathrm{~g} \mathrm{IV}$ and repeated twice postoperatively at eight hour intervals) with either gentamicin or a fluoroquinolone; the doses of the last two drugs are similar to those used in the above regimen

In contrast to the Surgical Care Improvement Project, which recommended routine redosing, the Medical Letter recommended redosing only for proce- dures lasting more than four hours and associated with major blood loss, according to the regimens cited above. Cefoxitin ( 1 to $2 \mathrm{~g} \mathrm{IV}$ ) Cefazolin (1 to 2 g IV) plus metronidazole (0.5 g IV) Ampicillin-sulbactam (3 g IV)

For patients allergic to penicillins or cephalosporins, clindamycin plus one of the following: the ciprofloxacin, levofloxacin, gentamicin, or aztreonam (Sullins and Abdel-Rahman, 2013).

\section{Other gastrointestinal surgery:}

The 2009 medical letter guidelines recommended antimicrobial prophylaxis in selected patients who undergo surgery at sites in the gastrointestinal tract other than the colon or rectum:

\section{Esophageal and gastro-duodenal:}

Prophylaxis in recommended in patients at increased risk, defined as esophageal obstruction, morbid obesity, and reductions in gastric acidity or gastrointestinal motility due to obstruction, hemorrhage, gastric ulcer, malignancy, or proton pump inhibitor therapy. The recommended antibiotics are cefazolin (1 to $2 \mathrm{~g} \mathrm{IV}$ ) or, in patients allergic to penicillins and cephalosporins, clindamycin plus one of the ciprofloxacin, levofloxacin, gentamicin, or aztreonam (Miyashita et al, 2013).

\section{Biliary tract surgery:}

Prophylaxis is recommended in patients at high risk for risk, defined as age greater than 70 years, acute cholecystitis, a nonfunctioning gallbladder, obstructive jaundice, and common bile duct stones. The recommended antibiotics are cefazolin ( 1 to $2 \mathrm{~g} \mathrm{IV}$ ) or, in patients allergic to penicillins and cephalosporins, clindamycin plus one of the following: ciprofloxacin, 
levofloxacin, gentamicin, or aztreonam.

Among patients undergoing endoscopic retrograde cholangiopancreatography (ERCP), routine antibiotic prophylaxis does not reduce sepsis/cholangitis (NNIS, 2002), even in patients thought to have biliary tract stones or distal bile duct stricture. However, prophylaxis is warranted in patients with cholangitis, biliary obstruction, and possibly incomplete drainage (van den Hazel et al, 1996).

On the other hand, randomized trials have shown that the risk of infection is low and that prophylaxis is not beneficial in low-risk patients undergoing elective laparoscopic cholecystectomy (Koc et al, 2003).

\section{Appendectomy:}

Antimicrobial therapy recommended in patients with non-perforated acute appendicitis for prophylaxis against wound infection and intra-abdominal abscess and with perforated appendicitis for therapy. The recommended antibiotics are cefoxitin ( 1 to $2 \mathrm{~g} \mathrm{IV}$ ), ampicillin/sulbactam (3 g IV), combination of cefazolin ( 1 to $2 \mathrm{~g} \mathrm{IV}$ ) plus metronidazole (500 $\mathrm{mg} \mathrm{IV})$, or, in patients allergic to penicillins and cephalosporins, clindamycin plus one of the ciprofloxacin, levofloxacin, gentamicin, or aztreonam (Chang et al, 2006).

\section{Mesh inguinal hernioplasty:}

There is growing evidence to support use of antimicrobial prophylaxis prior to hernioplasty. A 2007 Cochrane Review concluded that there was insufficient evidence in favor of or against administration of antimicrobial prophylaxis for elective inguinal hernia repairs
(Andersen et al, 2005). However, a subgroup-analysis of six randomized trials in which prosthetic material was used for hernia repair demonstrated that SSI rates decreased when antimicrobial prophylaxis was administered (OR 0.48, 95\% CI 0.27-0.85). Such antimicrobial prophylaxis is appropriate because of the known increased risk associated with implantation of prosthetic material. While many Medical Letter consultants do not recommend the routine use of surgical prophylaxis for these procedures, some favor prophylaxis for any procedure involving implantation of prosthetic material (Sanchez-Manuel et al, 2007).

\section{Pancreatic surgery:}

Pancreatic surgery frequently also involves biliary tract surgery. In such cases, existing guidelines for antimicrobial prophylaxis for biliary tract procedures can be applied to these cases. If biliary drainage procedures are performed endoscopically or percutaneously prior to pancreatic surgery, the risks of postoperative sepsis and surgical site infections are increased. Therefore, in such cases, antimicrobial prophylaxis prior to the pancreatic procedure should be expanded to cover microorganisms recovered from biliary drains. Similarly, antimicrobial prophylaxis may need to be adjusted to cover known or expected pathogens when pancreatic surgery is performed in patients who have pre-existing percutaneous devices placed to drain pancreatic or peripancreatic fluid collections. In the remainder of cases, a first- or second-generation cephalosporin as cefazolin or cefuroxime is an appropriate 
choice for pre-operative antimicrobial prophylaxis (Sanabria et al, 2007).

\section{Orthopedic surgery:}

Prophylaxis is warranted for total hip or knee replacement and other orthopedic procedures such as surgical repair of hip and other closed fractures and internal fixation by nails, plates, screws, or wires. A prospective, randomized trial of 437 patients undergoing diagnostic and operative arthroscopic surgery found only one infection and therefore no benefit from antibiotics (Dimick et al, 2000).

\section{Gynecologic or obstetric surgery:}

The prophylaxis is warranted for hysterectomy, surgical abortion, cesarean delivery, and certain other procedures (Kapan et al, 2013).

\section{Vascular surgery:}

Preoperative antimicrobial prophylaxis reduces the incidence of postoperative SSI in patients undergoing reconstructive surgery on the abdominal aorta, arterial surgery on the leg involving a groin incision, and amputation of the lower extremity due to critical limb ischemia. Many recommend prophylaxis for arterial surgery involving implantation of prosthetic material, such as grafts for vascular access for hemodialysis. The recommended drugs in patients undergoing vascular (arterial) surgery are cefazolin or cefuroxime and for patients with beta-lactam allergy, vancomycin (Regelsberger et al, 2011)

\section{Genitourinary surgery:}

Antimicrobial prophylaxis is warranted before urologic procedures when the urine culture is positive or not obtained or a urinary catheter is in place. Such patients should be treated to sterilize the urine before surgery or should receive a single preoperative dose of a drug active against the most likely pathogen. Antimicrobial prophylaxis also decreases the incidence of postoperative bacteriuria and sepsis in patients with sterile urine undergoing transurethral prostatectomy (Wieck et al, 1997), before transrectal prostatic biopsy, and implantation of a prosthesis, such as penile implant or artificial sphincter (Berry and Barratt, 2002).

The potential efficacy of prophylaxis was illustrated in a meta-analysis of 32 randomized trials in patients undergoing transurethral prostatectomy. Prophylaxis significantly reduced the rates of both postoperative bacteriuria ( 9 vs. $26 \%$ ) and clinical septicemia (0.7 vs. 4.4\%). Effective antibiotics included quinolones, cephalosporins, trimethoprim-sulfamethoxazole, and aminoglycosides. The Medical letter recommends ciprofloxacin $(500 \mathrm{mg}$ PO or $400 \mathrm{mg}$ IV) or trimethoprim-sulfamethoxazole (1 DS tablet orally) in these settings (Aron et al, 2000).

In the absence of an indwelling catheter, antimicrobial therapy can be discontinued promptly following the procedure. When an indwelling catheter must remain in place following prostatic resection some advocate continuing antimicrobial therapy until the catheter is removed. Cefazolin ( 1 to $2 \mathrm{~g}$ IV) should be given prior to open or laparoscopic genitourinary procedures; including percutaneous renal surgery or procedures with entry into the urinary tract (Nicolle et al, 2005). 


\section{Neurosurgery:}

Antimicrobial prophylaxis can reduce the incidence of infection, mostly due to $S$. aureus or S. epidermidis, after craniotomy and spinal surgery, including low-risk procedures (Dimick et al, 2000). Efficacy in clean neurosurgical operations was illustrated in a trial in which 846 patients were randomly assigned to cefazolin and gentamicin or no therapy (Young and Lawner, 1987). The treated group had a significantly lower rate of wound infection (1.0 vs. $3.6 \%$ ). All infections that occurred involved gram-positive organisms. Efficacy in spinal surgery was illustrated in a meta-analysis that evaluated six randomized trial involving 843 patients; four of the trials, including the previous trial, were general neurosurgical trials that included patients undergoing spinal surgery (Silverman et al, 2000). Although no individual trial demonstrated a statistically significant benefit from prophylaxis in patients undergoing spinal surgery, a significant reduction in infection rate was noted in the pooled analysis $(2.2$ vs. $5.9 \%$, pooled odds ratio $0.37,95 \%$ CI $0.17-0.78$ (Korinek et al, 2006). The recommended antibiotics are cefazolin ( 1 to $2 \mathrm{~g}$ IV) or, in selected patients, vancomycin (1 g IV). Indications for vancomycin are previous colonization with MRSA, hospitals where MRSA and/or coagulase-negative $S$, aureus are a common cause of postoperative infection, and allergy to the penicillins or cephalosporins. Clindamycin is an alternative to vancomycin in patients with beta-lactam allergy (Silverman et al, 2000).

\section{Head and neck surgery:}

The 2006 medical letter guidelines recommended antimicrobial prophylaxis for head and neck surgery involving an incision through the oral or pharyngeal mucosa (Sinha et al, 2013). The recommended antibiotics are cefazolin (1 to $2 \mathrm{~g} \mathrm{IV}$ ) or the combination of gentamicin $(1.5 \mathrm{mg} / \mathrm{kg} \mathrm{IV})$ plus clindamycin (600 to $900 \mathrm{mg} \mathrm{IV}$ ).

\section{Areas of controversy:}

Efficacy of antimicrobial prophylaxis is controversial in breast surgery, and other clean procedures. The 2009 medical letter guideline consultants do not recommend prophylaxis for such procedures due to the low rate of infection, low morbidity if infection does occur, and the potential adverse effect of antimicrobial therapy. Some recommend prophylaxis for procedures that involve placement of prosthetic material such as synthetic mesh or saline implants.

\section{Nasopharyngeal and oropharyngeal decontamination:}

The nasal carriage of $S$. aureus is a major risk factor for wound infection after cardiac surgery raised the possibility that nasopharyngeal and oropharyngeal decontamination might reduce the risk of postoperative infection. The drugs that have been evaluated include intranasal mupirocin and chlorhexidine gluconate (Kluytmans et al, 1995).

The efficacy of chlorhexidine gluconate decontamination was directly addressed in a randomized trial where 954 patients were randomly assigned to the chlorhexidine gluconate or placebo as nasal ointment and oropharyngeal rinse prior to elective cardiac surgery (mostly $\mathrm{CABG}$ and/or valve surgery). 
All patients received cefuroxime prophylaxis (Segers et al, 2006).

The following significant benefits were noted with chlorhexidine gluconate: A reduction in overall nosocomial infections (19.8 versus $26.2 \%$ with placebo) that was primarily due to reductions in deep surgical site infections $(1.9$ vs. $5.1 \%)$ and lower respiratory tract infections (9.3 vs. 15.8\%). There was no reduction in superficial surgical site infections (ie, those involving only the skin or subcutaneous tissue). This clinical benefit was associated with a greater reduction in $S$. aureus nasal carriage (57.5 vs. $18.1 \%$ ). Possible role of decontamination to eradicate MRSA carriage in hospitals where MRSA is a frequent cause of surgical site infection is discussed separately.

\section{Hair removal:}

Hair removal is commonly performed before many surgical procedures in order to provide the surgeon with a "clean" field and to prevent hair from falling into the surgical site. However, most studies have shown an increased risk for SSIs in patients undergoing preoperative hair removal (Mishriki et $a l, 1990)$. The rates of SSI were highest when shaving was performed compared to clipping the hair or use of depilatory creams (5.6 compared to 1.7 and $0.6 \%$, respectively). Scanning electron micrographs showed that razors caused gross skin cuts, clippers caused less injury, and depilatory agents caused no injury to the skin surface. Timing of hair removal is also important. The lowest rates of SSI were reported when hair was removed just prior to the surgical incision (Hamilton et al, 1977).

Several studies in neurosurgical patients have questioned the need for hair shaving. One study evaluated 1,038 cranial procedures, including craniotomy, stereotactic biopsy, ventriculoperitoneal shunt placement, aspiration of brain abscess, or resection of infected tissue, without hair removal, and compared the rate of infection to $1,038 \mathrm{pa}-$ tients whose heads were shaved. Patients had their hair washed with shampoo and $4 \%$ chlorhexidine within 24 hours of their operation. In the operating room, surgical site was scrubbed for 8 to 10 minutes with $4 \%$ chlorhexidine diluted with water, and then with $10 \%$ povidone-iodine solution. Prophylactic antibiotics were given for three days (Tang et al, 2001).

The investigators observed 13 postoperative wound infections $(1.3 \%)$, including nine deep $(0.9 \%)$ and four superficial $(0.4 \%)$ infections. There was no significant difference between the rates of infection in patients whose heads were shaven or not (12 of 980 versus 13 of 1038). Two small prospective trials in the highly selected, nonemergency cases have also supported not shaving hair in neurosurgical operations (Tang et al, 2001). A metaanalysis that examined 11 randomized controlled trials concluded that there was no difference in rate of SSIs in patients who have had hair removed prior to surgery and those who have not. If hair needs to be removed, patients should be clipped rather than shaved, as patients who were shaved were more likely to develop SSI (three 
trials, 3193 patients, relative risk 2.02, 95\% CI, 1.21-3.36 (Tanner et al, 2006). Hyperglycemia and diabetes control:

Hyperglycemia and diabetes have been identified as risk factors for deep sternal site infection after CABG. These studies have suggested that a preoperative blood glucose level of 200 $\mathrm{mg} / \mathrm{dL}$ or more (odds ratio [OR] 10.2) or postoperative hyperglycemia (OR, 2.0) was associated with an increased risk of SSIs (Latham et al, 2001).

A historic cohort study of 1574 patients who had undergone coronary artery bypass grafting between 1998 and 1999 demonstrated that each 50 $\mathrm{mg} / \mathrm{dL}(2.8 \mathrm{mmol} / \mathrm{L})$ blood glucose increase may be associated with higher mortality (odds ratio 1.37; 95\% CI, 0.98 to 1.92 ) and higher infection rate (odds ratio 1.23, 95\% CI 0.94 to 1.60 ). Each $50 \mathrm{mg} / \mathrm{dL}(2.8 \mathrm{mmol} / \mathrm{L})$ blood glucose increase was associated with a longer postoperative hospital stay by 0.76 days (95\% CI, 0.36 to 1.17 days) and increased hospitalization cost by $\$ 1769,95 \%$ CI, $\$ 928$ to $\$ 2610$ (Estrada et al, 2003).

A prospective study of 2467 consecutive diabetic patients who underwent open heart surgical procedures between 1987 and 1997 demonstrated that continuous intravenous insulin infusion led to a significant reduction in perioperative blood glucose levels and incidence of deep sternal wound compared with subcutaneous insulin injections, 0.8 vs 2.0\% (Furnary et al, 1999).

\section{Perioperative normothermia:}

Mild perioperative hypothermia may promote SSI by triggering thermoregulatory vasoconstriction that, in turn, may decrease subcutaneous oxygen tension. A study randomized 200 patients undergoing colorectal surgery to routine intraoperative thermal care (Hypothermia group) or the additional warming (Normothermia group) and found a lower rate of SSI in the latter group (19 vs 6\%). A similar randomized trial comparing systemic normothermia to conventional hypothermia was done in 1732 patients undergoing isolated coronary bypass surgery have found no difference in rates of sternal rewiring/debridement for dehiscence or infection (Kurz et al, 1996). But, hypothermia may protect tissue from ischemia by reducing oxygen consumption during surgery, an effect exploited during circulatory arrest in cardiac surgery. Despite this positive effect, most surgeons, anesthesiologists, and hospital epidemiologists acknowledge the benefit of perioperative normothermia in reducing the risk of SSI. A similar advantage to perioperative warming has been shown in one study of clean surgical procedures. Wound infection was significantly commonest among patients who were not warmed before surgery, 14 versus 5\% (Melling et al, 2001).

\section{Surveillance and infection control:}

An effective infection control program is an essential part of SSI prevention. The SENIC study reported that an effective infection-prevention program reduced the rate of SSIs by $41 \%$ in low-risk patients and by $35 \%$ in highrisk patients (Haley et al, 1985). Also, active surveillance and reporting of rates of SSIs to individual surgeons consistently resulted in a progressive 
decline in infection rates (Olson and Lee, 1990). Thus, SSI rates should be presented to surgeons and the chief of staff at regular intervals. These rates should be reported as surgeon-specific, service-specific, and hospital-wide and categorized within discrete risk index scores. Precautions need to be taken to ensure the confidentiality of these results. How such feedback brings about changes in surgeons' behavior is not known, but the benefits of its use are well supported. With the increased popularity of same day and ambulatory surgical procedures, the ability to identify and monitor SSI rates has decreased. This was illustrated in a study of post-discharge surveillance in 1324 patients undergoing CABG over a 27month period; 88 SSIs were identified, only $28 \%$ of which would have been detected during the initial hospitalization (Avato and Lai, 2002).

Efforts to track these infections have included patient and surgeon questionnaires and nurse observation of the wounds at the patient's home or during a routine postoperative visit. One report identified SSIs from an automated pharmacy system combined with computerized patient data; this methodology detected 96 SSIs in 4086 operations $(2.3 \%)$ for an overall sensitivity of $74 \%$, specificity of $98 \%$ and positive predictive value of $48 \%$. This performance was superior to patient or physician surveys (Sands et al, 1999). A second study directly compared this type of surveillance to hospital-based surveillance in determining SSIs in patients who had undergone CABG. The health plan and automated pharmacy information detected more patients with SSI than hospital surveillance including pre-discharge infections. One or both methods identified 167 infected patients; the sensitivity of surveillance based upon health plan data was $72 \%$ compared with $50 \%$ for hospital surveillance (Sands et al, 2003). Prospective cohort study from a tertiary hospital in Spain utilized telephone followup a month after surgery to successfully contact $98 \%$ of 1506 patients. The investigators suggested that predictors of SSI were not the same for patients in the immediate postoperative period while still hospitalized and for those who developed infection following discharge. Only predictors of post-discharge SSI were body mass index, age, \& improperly timed prophylactic antibiotics (Delgado-Rodriguez et al, 2001).

\section{Healthcare improvement campaign:}

Institute for Healthcare Improvement (IHI) is a nonprofit organization leading the improvement of health care throughout the world. The 100,000 lives campaign was an initiative to engage United States hospitals in a commitment to implement changes in care proven to improve patient care and prevent avoidable deaths. The six initiatives in the 100,000 lives campaign were included in the newer 5 million lives campaign along with specific recommendations to reduce the incidence of surgical site infections by promoting the following modalities adopted earlier by surgical infection prevention and surgical care improvement projects.

\section{Antibiotics appropriate use:}

Antibiotics should be administered within one hour before surgical 
incision, the choice of prophylactic antibiotics should be consistent with national guidelines and prophylactic antibiotics should be discontinued within 24 hours after surgery.

\section{Appropriate hair removal:}

Remove all razors from entire hospital, work with purchasing department so that razors are no longer purchased, use reminders, and educate patients not to self-shave preoperatively. Maintenance of postoperative glucose control for major cardiac surgery patientsImplement a glucose control protocol, regularly check preoperative glucose levels on all patients, and assign responsibility and accountability for blood glucose monitoring and control.

\section{Establishment of perioperative nor-} mothermia:

Use warmed, forced-air blankets preoperatively during surgery and in the Post Anesthesia Care Unit (PACU), use warmed IV fluids, increase the ambient temperature in the operating room, use warming blankets under patients on the operating table, and use hats and booties on patients preoperatively. Hospitals that collaborated by instituting programs to utilize the above four measures reported a mean $27 \%$ reduction in SSI rates (from 2.3 to $1.7 \%$ in the first versus last three months of the study (Wu, 2013).

\section{References}

Ahmed, B, Martinez, JD, Schevchuck, A, Ahmed, S, Schrader, R, et al, 2012: Appropriate timing of nitroglycerin prior to intravascular ultrasound. J. Invasive Cardiol. 24, 9:422-6. Altemeier, WA, Burke, JF, Pruitt, BA, Sandusky, WR, 1984: Manual on
Control of Infection in Surgical Patients. JB Lippincott, Philadelphia.

Andersen, BR, Kallehave, FL, Andersen, HK, 2005: Antibiotics versus placebo for prevention of postoperative infection after appendicectomy. Cochrane Database Syst. Rev.: CD001439.

Aron, M, Rajeev, T, Gupta, N, 2000: Antibiotic prophylaxis for transrectal needle biopsy of the prostate: a randomized controlled study. BJU Int. 85: 682.

Avato, JL, Lai, KK, 2002: Impact of post-discharge surveillance on surgicalsite infection rates for coronary artery bypass procedures. Infect. Cont. Hosp. Epidemiol. 23:364-9.

Aznar, R, Mateu, M, Miro, JM, et al, 1991: Antibiotic prophylaxis in noncardiac thoracic surgery: cefazolin versus placebo. Eur. J. Cardiothorac. Surg. 5:515.

Baddour, LM, Bettmann, MA, Bolger, AF, et al, 2003: Nonvalvular cardio-vascular device-related infections. Circulation 108:2015.

Bekar, A, Korfali, E, Dogan, S, et al, 2001: Effect of hair on infection after cranial surgery. Acta Neurochi. (Wien) 143:533-40.

Berry, A, Barratt, A, 2002: Prophylactic antibiotic use in transurethral prostatic resection: a meta-analysis. J. Urol. 167:571.

Bolon, MK, Morlote, M, Weber, SG, et al, 2004: Glycopeptides are no more effective than beta-lactam agents for prevention of surgical site infection after cardiac surgery: a meta-analysis. Clin. Infect. Dis. 38:1357.

Boyce, JM, Pittet, D, 2002: Guideline 
for hand hygiene in health-care settings: recommendations of the healthcare infection control practices advisory committee and HICPAC/SHEA/ AP IC/IDSA Hand Hygiene Task Force. Infect. Cont. Hosp. Epidemiol. 23:S3 Bratzler, DW, Houck, PM, 2002: Antimicrobial prophylaxis for surgery: An advisory statement from the national surgical infection prevention project. Clin. Infect. Dis. 38:1706. Bratzler, DW, Houck, PM, Richards, C, et al, 2005: Use of antimicrobial prophylaxis for major surgery: baseline results from the national surgical infec-tion prevention project. Arch. Surg. 140:174.

Bratzler, DW, Hunt, DR, 2006: The surgical infection prevention and surgical care improvement projects: National initiatives to improve outcomes for patients having surgery. Clin. Infect. Dis. 43:322.

CDC, 1992a: The surgical infection society. Infect. Cont. Hosp. Epidemiol. 13: 599-608.

CDC, 1992b: A modification of CDC definitions of surgical wound infections. Am. J. Infect. Control 20:271.

Chang, WT, Lee, KT, Chuang, SC, et al, 2006: The impact of prophylactic antibiotics on postoperative infection complication in elective laparoscopic cholecystectomy: a prospective randomized study. Am. J. Surg.191:721.

Cruse, PJ, Ford, R, 1980: The epidemiology of wound infection: A 10-year prospective study of 62,939 wounds. Surg. Clin. North Am. 60:27.

Curtis, JJ, Boley, TM, Walls, J, et al, 1993: Randomized, prospective comparison of first- and second-generation cephalosporins as infection prophylaxis for cardiac surgery. Am. J. Surg. 166: 734-9.

Delgado-Rodriguez, M, Gomez-Ortega, A, Sillero-Arenas, M, Llorca, J, 2001: Epidemiology of surgical-site infections diagnosed after hospital discharge: a prospective cohort study. Infect. Control Hosp. Epidemiol. 22: 24.

Dellinger, EP, 2007: Prophylactic antibiotics: administration and timing before operation are more important than administration after operation. Clin. Infect. Dis. 44:928.

Dellinger, EP, Hausmann, SM, Bratzler, DW, et al, 2005: Hospitals collaborate to decrease surgical site infections. Am. J. Surg. 190:9.

Dimick, JB, Lipsett, PA, Kostuik, JP, 2000: Spine update: antimicrobial prophylaxis in spine surgery: basic principles and recent advances. Spine 25: 2544.

DiPiro, JT, Vallner, JJ, Bowden, TA $\mathrm{Jr}$, et al, 1985: Intraoperative serum and tissue activity of cefazolin and cefoxitin. Arch. Surg. 120:829.

Doebbeling, BN, Pfaller, MA, Kuhns, KR, et al, 1990: Cardiovascular surgery prophylaxis: A randomized, controlled comparison of cefazolin and cefuroxime (see comments). J. Thorac. Cardiovasc. Surg. 99:981.

Edwards, PS, Lipp, A, Holmes, A, 2004: Preoperative skin antiseptics for preventing surgical wound infections after clean surgery. Cochrane Database Syst. Rev.:CD003949

Estrada, CA, Young, JA, Nifong, L W, Chitwood, WR, 2003: Outcomes and perioperative hyperglycemia in patients with or without diabetes mellitus under-going coronary artery bypass 
grafting. Ann. Thorac. Surg. 75:1392.

Farrington, M, Webster, M, Fenn, A, Phillips, I, 1985: Study of cardiothoracic wound infection at St. Thomas' Hospital. Br. J. Surg. 72:759.

Finkelstein, R, Rabino, G, Mashiah, $\mathrm{T}$, et al, 2002: Vancomycin vs cefazolin prophylaxis for cardiac surgery in setting of a high prevalence of methicillin-resistant staphylococcal infections. J. Thorac. Cardiovasc. Surg. 123: 326.

Furnary, AP, Zerr, K, Grunkemeier, G, Starr, A, 1999: Continuous intravenous insulin infusion reduces incidence of deep sternal wound infection in diabetic patients after cardiac surgical procedures. Ann. Thorac. Surg. 67: 352.

Garey, KW, Dao, T, Chen, H, et al, 2006: Timing of vancomycin prophylaxis for cardiac surgery patients and the risk of surgical site infections. J. Antimicrob. Chemother. 58:645-9.

Garey, KW, Lai, D, Dao-Tran, T, et al, 2008: Interrupted time series analysis of vancomycin compared to cefuroxime for surgical prophylaxis in patients undergoing cardiac surgery. Antimicrob. Agents Chemother. 52: 44652.

Haley, RW, Culver, DH, White, J, et $\boldsymbol{a l}$, 1985: Efficacy of infection surveillance and control programs in preventing nosocomial infections in US hospitals. Am. J. Epidemiol. 121:182-90.

Hamilton, HW, Hamilton, KR, Lone, FJ, 1977: Preoperative hair removal. Can. J. Surg. 20:269.

Harbarth, S, Samore, MH, Lichtenberg, D, Carmeli, Y, 2000: Prolonged antibiotic prophylaxis after cardiovas- cular surgery and its effect on surgical site infections and antimicrobial resistance. Circulation 101:2916.

Hayek, LJ, Emerson, JM, Gardner, AM, 1987: A placebo-controlled trial of the effect of two preoperative baths or showers with chlorhexidine detergent on postoperative wound infection rates. J. Hosp. Infect. 10:165-9.

Itani, KM, Wilson, SE, Awad, SS, et al, 2996: Ertapenem versus cefotetan prophylaxis in elective colorectal surgery. N. Engl. J. Med. 355:2640.

Jethwa, P, Breuning, E, Bhati, C, et al, 2007: The microbiological impact of pre-operative biliary drainage on patients undergoing hepato-biliary-pancreatic (HPB) surgery. Aliment. Pharmacol. Ther. 25:1175-82.

Kapan, S, Bozkurt, MA, Turhan, N, Gönenç, M, Alış, H, 2013: Management of acute appendicitis in pregnancy. Ulus. Travma. Acil. Cerrahi Derg. 19, 1:20-4.

Kernodle, DS, Classen, DC, Burke, JP, Kaiser, AB, 1990: Failure of cephalo-sporins to prevent Staphylococcus aureus surgical wound infections. JAMA 263:961.

Kluytmans, JA, Mouton, JW, Ijzerman, EP, et al, 1995: Nasal carriage of Staphylococcus aureus as a major risk factor for wound infections after cardiac surgery. J. Infect. Dis. 171:216.

Koc, M, Zulfikaroglu, B, Kece, C, Ozalp, N, 2003: A prospective randomized study of prophylactic antibiotics in elective laparoscopic cholecystectomy. Surg. Endosc. 17:1716-9.

Korinek, AM, Baugnon, T, Golmard, JL, et al, 2006: Risk factors for adult nosocomial meningitis after cranioto- 
my: role of antibiotic prophylaxis. Neurosurg. 59:126-32.

Kreter, B, Woods M, 1992: Antibiotic prophylaxis for cardiothoracic operations: Meta-analysis of thirty years of clinical trials. J. Thorac. Cardiovasc. Surg. 104:590.

Kurz, A, Sessler, DI, Lenhardt, R, 1996: Perioperative normothermia to reduce the incidence of surgical-wound infection and shorten hospitalization: Study of wound infection and temperature group. N. Engl. J. Med. 334: 1209. Latham, R, Lancaster, AD, Covington, JF, et al, 2001: The association of diabetes and glucose control with surgical-site infections among cardiothoracic surgery patients. Infect. Cont. Hosp. Epidemiol. 22:607-12.

Lewis, RT, 2002: Oral versus systemic antibiotic prophylaxis in elective colon surgery: a randomized study and metaanalysis send a message from 1990s. Can. J. Surg. 45:173.

Mangram, AJ, Horan, TC, Pearson, ML, et al, 1999: Guideline for prevention of surgical site infection: Hospital Infection Control Practices Advisory Committee. Infect. Control Hosp. Epidemiol. 20:250-60.

McDonald, M, Grabsch, E, Marshall, C, Forbes, A, 1998: Single-versus multiple-dose antimicrobial prophylaxis for major surgery: a systematic review. Aust. N. Z. J. Surg. 68:388.

Melling, AC, Ali, B, Scott, EM, Leaper, DJ, 2001: Effects of preoperative warming on the incidence of wound infection after clean surgery: a randomized controlled trial. Lancet 358:876.
Mishriki, SF, Law, DJ, Jeffery, PJ, 1990: Factors affecting the incidence of postoperative wound infection. J. Hosp. Infect.16:223.

Miyashita, T, Shah, FA, Miwa, K, Sasaki, S, et al, 2013: Impact of inflammation-metaplasiaadenocarcinoma sequence and prevention in surgical rat models. Digestion 87, 1:6-11.

Namias, N, Harvill, S, Ball, S, et al, 1999: Cost and morbidity associated with antibiotic prophylaxis in the ICU. J. Am, Coll. Surg. 188:225.

Nicolle, LE, Bradley, S, Colgan, R, et al, 2005: Infectious Diseases Society of America guidelines for the diagnosis and treatment of asymptomatic bacteriuria in adults. Clin. Infect. Dis. 40:643.

NNIS, 2002: National Nosocomial Infections Surveillance: System Report, data summary from January 1992 to June 2002. Am. J. Infect. Cont. 30:458.

Olson, MM, Lee JT, Jr, 1990: Continuous, 10-year wound infection surveillance: Results, advantages, and unanswered questions. Arch. Surg. 125: 794.

Parienti, JJ, Thibon, P, Heller, R, et $\boldsymbol{a l}$, 2002: Hand-rubbing with an aqueous alcoholic solution vs traditional surgical hand-scrubbing \& 30-day surgical site infection rates: a randomized equivalence study. JAMA 288: 722.

Pearlman, MD, Pierson, CL, Faix, RG, 1998: Frequent resistance of clinical group B streptococci isolates to clindamycin and erythromycin. Obstet. Gynecol. 92, 2:258-61

Perencevich, EN, Sands, KE, Cosgrove, SE, et al, 2003: Health and economic impact of surgical site infec- 
tions diagnosed after hospital discharge. Emerg. Infect. Dis. 9:196.

Periti, P, Stringa, G, Mini, E, 1999: Comparative multicenter trial of teicoplanin versus cefazolin for antimicrobial prophylaxis in prosthetic joint implant surgery: Italian study group for antimicrobial prophylaxis in orthopedic surgery. Eur. J. Clin. Microbiol. Infect. Dis. 18:113.

Pollard, JP, Hughes, SP, Scott, JE, et al, 1979: Antibiotic prophylaxis in total hip replacement. Br. Med. J. 1:707.

Pons, VG, Denlinger, SL, Guglielmo, BJ, et al, 1993: Ceftizoxime versus vancomycin and gentamicin in neurosurgical prophylaxis: A randomized, prospective, blinded clinical study. Neurosurgery 33:416.

Regelsberger, J, Elsayed, A, Matschke, J, Lindop, G, Grzyska, U, et al, 2011: Diagnostic and therapeutic considerations for "mycotic" cerebral aneurysms: 2 case reports and review of the literature. Cent. Eur. Neurosurg. 72, 3:138-43.

Rotter, ML, Hirschl, AM, Koller, W, 1988: Effect of chlorhexidinecontaining detergent, non-medicated soap or isopropanol and the influence of neutralizer on bacterial pathogenicity. J. Hosp. Infect. 11:220-8.

Saginur, R, Croteau, D, Bergeron, $M$ G, 2000: Comparative efficacy of teico-planin and cefazolin for cardiac operation prophylaxis in 3027 patients. J. Thorac. Cardiovasc. Surg. 120:1120. Sanabria, A, Dominguez, LC, Valdivieso, E, Gomez, G, 2007: Prophylactic antibiotics for mesh inguinal hernioplasty: a meta-analysis. Ann. Surg. 245:392.
Sanchez-Manuel, FJ, Lozano-Garcia, J, Seco-Gil, JL, 2007: Antibiotic prophylaxis for hernia repair. Cochrane Database Syst. Rev.:CD003769.

Sands, K, Vineyard, G, Livingston, J, et al, 1999: Efficient identification of postdischarge surgical site infections: Use of automated pharmacy dispensing information, administrative data, and medical record information. J. Infect. Dis. 179:434.

Sands, KE, Yokoe, DS, Hooper, DC, et al, 2003: Detection of postoperative surgical-site infections: Comparison of health plan-based surveillance with hospital-based programs. Infect. Control Hosp. Epidemiol. 24:741-8.

Segers, P, Speekenbrink, RG, Ubbink, DT, et al, 2006: Prevention of noso-comial infection in cardiac surgery by decontamination of the nasopharynx and oropharynx with chlorhexidine gluconate: a randomized controlled trial. JAMA 296:2460.

Selwyn, S, Ellis, H, 1972: Skin bacteria and skin disinfection reconsidered. Br. Med. J. 1:136.

Sexton, DJ, 2006: Carbapenems for surgical prophylaxis? N. Engl. J. Med. 355:2693.

Silverman, N, Morgan, M, Nichols, W, 2000: Antibiotic resistance patterns of group B streptococcus in antenatal genital cultures. J. Reprod. Med. 45, 12:979-82.

Sinha, R, Das, S, Banerjee, P, Halder, A, Dutta, M, 2013: Huge hibernoma of the neck with extension into the mediastinum. Ear Nose Throat J. 92, 4/5:E18-21.

Song, F, Glenny, A, 1998: Antimicrobial prophylaxis in colorectal surgery: a 
systematic review of randomized controlled trials. Br. J. Surg. 85:1232.

Sullins, AK, Abdel-Rahman, S, 2013: pharmacokinetics of antibacterial agents in the CSF of children and adolescents. Paediatr. Drugs 15, 2:93-117.

Tang, K, Yeh, JS, Sgouros, S, 2001: Influence of hair shaves on infection rate in neurosurgery: A prospective study. Pediatr. Neurosurg. 35: 13-9.

Tanner, J, Woodings, D, Moncaster, K, 2006: Preoperative hair removal to reduce surgical site infection. Cochrane Database Syst. Rev.:CD004122;

Tanner, J, Swarbrook, S, Stuart, J, 2008: Surgical hand antisepsis to reduce surgical site infection. Cochrane Database Syst. Rev.:CD004288.

Townsend, TR, Reitz, BA, Bilker, WB, Bartlett, JG, 1993: Clinical trial of cefamandole, cefazolin, and cefuroxime for antibiotic prophylaxis in cardiac operations. J. Thorac. Cardiovasc. Surg. 106:664.

Trivedi, M, Patel, V, Soman, R, Rodriguez, C, Singhal, T, 2012: Outcome of treating ESBL infections with carbapenems vs. non carbapenem antimicrobials. J. Assoc. Physicians India 60:28-30.

van den Hazel, SJ, Speelman, P, Dankert, J, et al, 1996: Piperacillin to prevent cholangitis after endoscopic retrograde cholangio-pancreatography: A randomized, controlled trial. Ann. Int. Med. 125:442.

van Kasteren, ME, Mannien, J, Ott, A, et al, 2007: Antibiotic prophylaxis and the risk of surgical site infections following total hip arthroplasty: Timely administration is the most important fa- ctor. Clin. Infect. Dis. 44:921.

Vuorisalo, S, Pokela, R, Syrjala, H, 1998: Comparison of vancomycin and cefuroxime for infection prophylaxis in coronary artery bypass surgery. Infect Control Hosp. Epidemiol. 19:234-40.

Weber, W, Marti, W, Zwahlen, M, et al, 2008: Timing of surgical antimicrobial prophylaxis. Ann. Surg. 247:918.

Webster, J, Osborne, S, 2006: Preoperative bathing or showering with skin antiseptics to prevent surgical site infection. Cochrane Database Syst. Rev.: CD004985.

Wieck, JA, Jackson, JK, O'Brien, TJ, et al, 1997: Efficacy of prophylactic antibiotics in arthroscopic surgery. Orthopedics 20:133.

Wong-Beringer, A, Corelli, RL, Schrock, TR, Guglielmo, BJ, 1995: Influence of timing of antibiotic administration on tissue concentrations during surgery. Am. J. Surg. 169:379-83.

Wu, X, 2013: The safe and efficient use of forced-air warming systems. AORN J. 97, 3:302-8.

Young, RF, Lawner, PM, 1987: Perioperative antibiotic prophylaxis for prevention of postoperative neurosurgical infections: A randomized clinical trial. J. Neurosurg. 66:701-8.

Zanetti, G, Giardina, R, Platt, R, 2001: Intraoperative redosing of cefazolin and risk for surgical site infection in cardiac surgery. Emerg. Infect. Dis. $7: 828$.

Zmora, O, Wexner, S, Hajjar, L, et$\boldsymbol{a l}, \mathbf{2 0 0 3}$ : Trends in preparation for colorectal surgery: survey of the members of the American society of colon and rectal surgeons. Am. Surg. 69:150-8. 